\title{
Ovarian endometriomas and IVF: a retrospective case-control study
}

\author{
Francesca Bongioanni ${ }^{1}$, Alberto Revelli ${ }^{2}$, Gianluca Gennarelli ${ }^{2}$, Daniela Guidetti', Luisa Delle Delle Piane ${ }^{2}$ and \\ Jan Holte ${ }^{3}$
}

\begin{abstract}
We performed this retrospective case-control study analyzing 428 first-attempt in vitro fertilization (IVF) cycles, among which 254 involved women with a previous or present diagnosis of ovarian endometriosis. First, the results of these 254 cycles were compared with 174 cycles involving patients with proven non-endometriotic tubal infertility having similar age and body mass index. Women with ovarian endometriosis had a significantly higher cancellation rate, but similar pregnancy, implantation and delivery rates as patients with tubal infertility. Second, among the women with ovarian endometriosis, the women with a history of laparoscopic surgery for ovarian endometriomas prior to IVF and no visual endometriosis at ovum pick-up $(n=112)$ were compared with the nonoperated women and visual endometriomas at ovum pick-up $(n=142)$. Patients who underwent ovarian surgery before IVF had significantly shorter period, lower antral follicle count and required higher gonadotropin doses than patients with non-operated endometriomas. The two groups of women with a previous or present ovarian endometriosis did, however, have similar pregnancy, implantation and live birth rates. In conclusion, ovarian endometriosis does not reduce IVF outcome compared with tubal factor. Furthermore, laparoscopic removal of endometriomas does not improve IVF results, but may cause a decrease of ovarian responsiveness to gonadotropins.
\end{abstract}

\section{Background}

Laparoscopic stripping of endometriomas before IVF/ ICSI treatment in order to improve its outcome is widespread in everyday clinical practice. This procedure is, however, not based on clinical evidence[1-3]. Although a previous metanalysis [4], showed reduced pregnancy rates after IVF in women with ovarian endometriosis compared to patients undergoing IVF for other indications [5], other later studies could not confirm this finding [6-9]. When the influence of the surgical removal of endometriomas on IVF outcome was studied, operated patients obtained IVF results comparable to women without previous surgical intervention [10-15]. Furthermore, some results suggest that the surgical intervention may have a negative effect on ovarian reserve [12,16-18] and hence putatively compromise treatment outcome and long-term fertility.

\footnotetext{
* Correspondence: fertisave@yahoo.com

${ }^{2}$ Reproductive Medicine and IVF Unit, Department of Obstetrical and Gynecological Sciences, University of Torino, OIRM-S, Anna Hospital, Torino, Italy

Full list of author information is available at the end of the article
}

Thus, the possible impact of ovarian endometriosis on ART results remain a controversial issue. The aim of the present study was to retrospectively analyze a large number of IVF/ICSI cycles and to evaluate the treatment outcome (a) in patients with a diagnosis of ovarian endometriosis in comparison to that in patients with tubal infertility and (b) in women with a history of laparoscopic removal of ovarian endometriomas prior to IVF compared with the outcome in women without previous surgical intervention and a visual endometrioma at OPU.

\section{Methods}

\section{Patients}

A total number of 8623 first-attempt IVF cycles performed in three IVF units were retrospectively analyzed. In total, 254 cycles were found to involve women diagnosed with ovarian endometriosis. Of these, 142 women had never undergone any ovarian surgery and displayed one or more in situ ovarian endometriomas of small to medium size $(<=6 \mathrm{~cm}$ in diameter; Group A), and 112 women underwent IVF after the laparoscopic removal of 
one or more endometrioma(s) of comparable size $(<=6$ $\mathrm{cm}$ in diameter) by the classical "stripping" technique (Group B). These women did not have visual endometriomas at the time of OPU. The proportion of patients with bilateral endometriomas was $14.1 \%$ in Group A (20 out of 142 ) and $19.6 \%$ in group B (22 out of 112), a difference not statistically significant. In case of bilateral or multiple endometriomas, their diameter was summed: in all cases, the total diameter did not exceed $6 \mathrm{~cm}$. Endometriosis patients in Group B were operated because they were symptomatic, whereas those in Group A were not operated before IVF because they were no or less symptomatic, although they had endometriomas of similar size.

In all patients submitted to laparoscopic ovarian resection, the diagnosis of ovarian endometriosis was histologically confirmed. In women who had not been operated before IVF (Group A), the diagnosis of ovarian endometriosis was based on transvaginal ultrasound (evidence of an adnexal mass with diffuse low level echoes without neoplastic or acute hemorrhage features; [19]) and the AFS stage was estimated to be II-III in all cases. None of the patients operated for ovarian endometriosis received $\mathrm{GnRH}$-agonists or other medical treatments prior to or after operation.

The control group consisted of 174 women who underwent IVF treatment during the same time period, with laparoscopically diagnosed tubal factor and without any evidence of ovarian endometriosis. These women covered similar ranges of age and BMI as the endometriosis patients (Group C).

\section{IVF procedure}

Ovarian stimulations were conducted with daily subcutaneous injections of individual starting doses of $\mathrm{rFSH}$ (Follitropin alfa (Merck-Serono, Geneva, Switzerland) or Follitropin beta (Organon, Oss, the Netherlands) or hMG (Meropur/Menopur, Ferring, Switzerland) at appropriate doses (100-450 IU), estimated according to the woman'sage, the antral follicle count and the basal (day 3) FSH. The long GnRH-agonist down-regulation protocol was used (Nafarelin-Pfizer Inc., New York, USA) $400 \mathrm{mg}$ nasally twice daily, or buserelin (SanofiAventis, Paris, France) 0.3 mg nasally four times daily); in both cases half the dose was administered during ovarian stimulation. Ovarian response to gonadotropins was monitored by transvaginal ultrasound plus serum E2 measurement every third day from stimulation day 7. Ovulation was triggered by injecting 10,000 IU hCG s.c. when the leading follicle reached $18 \mathrm{~mm}$, with appropriate serum E2 levels. Transvaginal ultrasound-guided oocyte aspiration (OPU) was performed approximately 36 hours after hCG injection under local anaesthesia (paracervical block). Either IVF or
ICSI was performed according tothe clinical indication. After cultivation, embryos were transferred on Day 2 or 3 after ovum pick-up (OPU). Luteal phase support was given vaginally to all patients for 2 weeks from embryo transfer (progesterone vagitories (Apoteket AB, Stockholm, Sweden) $1200 \mathrm{mg}$ or gel (Merck-Serono, Geneva, Switzerland) $180 \mathrm{mg}$ daily). Pregnancy was defined as the visualization of a gestational sac at vaginal ultrasound investigation in gestational week 7. All data were de-identified ahead of analysis. The study did not in any way alter our routine IVF/ICSI protocols, nor did it involve any additional intervention at treatment. All data were prospectively collected with the intention to evaluate impact on treatment outcome.

\section{Ovarian surgery technique}

A four-port laparoscopy technique was used: an $11 \mathrm{~mm}$ trocar was inserted through a short umbilical incision and connected to a video monitor (WideVieuw ${ }^{\mathrm{TM}} \mathrm{HD}$ Karl Storz Endoscope); two additional lateral $5 \mathrm{~mm}$ operating ports and a central sovrapubic $5-10 \mathrm{~mm}$ operating port were also inserted. The pneumo-peritoneum was achieved by inflating $\mathrm{CO} 2(10 \mathrm{mmHg})$.

To excide endometriomas, an incision was performed at the antimesenteric site of the affected ovary using bipolar cautery; then, the endometrioma was drained with aspiration and the pseudo-capsule was dissected by gentle traction and countertraction using two $5 \mathrm{~mm}$ grasping forceps ("stripping"). The bleeding at the stripping site was stopped by bipolar cautery, only when necessary and very carefully in order to avoid unnecessary thermal damage to the healthy ovarian tissue.

\section{Assessment of ovarian sensitivity to FSH}

To assess ovarian sensitivity to FSH, the ratio between the number of retrieved oocytes and the number of FSH IU (x100) was calculated. This variable was defined "ovarian sensitivity", as it shows the effective ovarian response to FSH stimulation, independently on the total amount of administered FSH. Patients with more abundant ovarian follicular reserve tend to display higher ovarian sensitivity to exogenous FSH, whereas women with a low ovarian reserve usually have a lower ovarian sensitivity.

\section{Statistical analysis}

Data are expressed as the mean \pm SEM or as percentages when required. Statistical comparisons among groups were performed using the Fisher exact test, Yeats' corrected $\chi^{2}$, Wilcoxon's test or Student's t test, as appropriate. The JMP software was used for statistical elaboration. Significance was defined as a p value $<0.05$. 


\section{Results}

IVF outcome in patients with ovarian endometriosis vs. patients with tubal factor

The basal characteristics of patients with ovarian endometriosis (Group A), previously operated of endometrioma excision (Group B) and tubal infertility (Group C, controls) are shown in Table 1.

The groups were comparable for age, BMI, infertility duration, smoking habits, mean period, prevalence of associated male factor, and basal (day 3) FSH levels. The basal antral follicle count was significantly lower in women previously submitted to the excision of ovarian endometriosis (Group B) than in women with ovarian endometrioma(s) (Group A) or tubal factor infertility (Group C).

The outcome of IVF in patients affected by ovarian endometriosis versus woman with tubal factor is shown in Table 2. Women with ovarian endometriosis showed a significantly higher cancellation rate, but those who completed ovarian stimulation had a similar yield of oocytes at OPU and a comparable ovarian sensitivity compared to control subjects. Fertilization rates were similar, but women with endometriosis showed a lower percentage of cycles with complete failure of fertilisation. Overall, the implantation, pregnancy and live-birth rates per started cycle, OPU, and ET were similar in the groups.

IVF outcome in patients with in situ ovarian endometrioma(s) vs. patients previously operated for ovarian endometrioma(s)

The clinical characteristics of patients having one or more in situ ovarian endometriomas at the time of IVF

Table 1 Clinical characteristics of patients having one or more in situ endometrioma(s) at the time of IVF (Group A) vs. those previously operated for laparoscopic endometrioma(s) removal (Group B) vs. women with tubal infertility (Group C, controls)

\begin{tabular}{lcccc}
\hline & Group A & Group B & Group C & p \\
\hline Patients & 142 & 112 & 174 & \\
Age (yrs) & $33.8 \pm 3.1$ & $33.6 \pm$ & $34.0 \pm$ & $\mathrm{ns}$ \\
& & 4.4 & 3.1 & \\
BMI (kg/m ${ }^{2}$ ) & $22.7 \pm 3.2$ & $22.4 \pm$ & $23.1 \pm$ & $\mathrm{ns}$ \\
& & 3.2 & 3.3 & \\
Smoke (\%) & 11.8 & 16.1 & 15.3 & $\mathrm{~ns}$ \\
Mean period (days) & $28.8 \pm 4.0$ & $27.2 \pm$ & $28.5 \pm$ & $<0.005^{1}$ \\
& & 4.1 & 3.1 & $<0.004^{2}$ \\
Infertility duration (years) & $4.0 \pm 2.5$ & $3.9 \pm 2.9$ & $3.6 \pm 0.3$ & $\mathrm{~ns}$ \\
Associated male factor & 13.8 & 19.1 & 13.7 & $\mathrm{~ns}$ \\
(\%) & & & & \\
Antral follicle count & $16.9 \pm$ & $11.7 \pm$ & $16.6 \pm$ & $<$ \\
& 11.1 & 9.4 & 9.5 & $0.001^{1,2}$ \\
FSH day 3 level (U/l) & $7.2 \pm 3.9$ & $7.9 \pm 4.2$ & $6.6 \pm 3.5$ & $\mathrm{~ns}$ \\
\hline
\end{tabular}

${ }^{1}$ Group A vs Group B, ${ }^{2}$ Group B vs Group C, ns: not significant.
(Group A) and of women who had been previously operated for endometrioma removal (Group B) are shown in Table 1. Operated women had a shorter mean period and lower antral follicle counts than women with in situ endometrioma(s).

Women with previous ovarian surgery required higher total FSH doses than patients without previous surgery, but had comparable numbers of oocytes at OPU (Table $2)$. Thus, ovarian sensitivity was lower in operated women. The implantation rate, pregnancy rate and livebirth rate per started cycle, OPU, and ET were similar in the two groups.

\section{Discussion}

The present study retrospectively analyzed a large cohort of patients undergoing IVF in the years 20042009 , identifying 254 patients with a previous or present diagnosis of ovarian endometriosis. With the limitations of a retrospective study (although on a remarkably large number of observations), our results suggest that previous or present ovarian endometriosis does not impair success rates at IVF/ICSI, and that ovarian surgery for endometriosis does not result in improved ART outcome, but, on the contrary, may compromise ovarian reserve.

Laparoscopic stripping of ovarian endometriomas as an intervention to improve fertility is a widespread clinical practice, not only to improve natural fertility, but also to improve IVF outcome. This surgical strategy is used because of the following reasons: a) older studies suggested that patients with ovarian endometriosis had poorer IVF outcome than women with other infertility causes; b) some data suggest that spontaneous fecundity may improve after laparoscopic cystectomy [1]; c) some argue that puncturing an endometrioma during oocyte retrieval could spread endometriotic cells in the abdominal cavity or cause a pelvic infection.

The risk of complications linked to the puncture of ovarian endometriomas is, however, minimal: infections have been reported only sporadically [20,21], and indeed a study in which ovarian endometriomas were intentionally punctured and aspirated at the time of oocyte retrieval reported no complications [22]. Furthermore, aspiration of endometriomas followed by local injection of methotrexate [23] or alcoholic solutions $[24,25]$ is considered a therapeutic option for ovarian endometriosis.

As for IVF outcome in women with a diagnosis of ovarian endometriosis, the published data exhibit varying results. A meta-analysis from 2002 including 22 studies showed a reduced pregnancy rate after IVF in women with endometriosis compared to treatments in women with other infertility causes, and also showed a linear (inverse) relationship between the stage of the 
Table 2 IVF outcome of patients having one or more in situ endometrioma(s) at the time of IVF (Group A) vs. those previously operated for laparoscopic endometrioma(s) removal (Group B) vs. woman with tubal infertility (Group C, controls)

\begin{tabular}{|c|c|c|c|c|}
\hline & Group A & Group B & Group C & $\mathrm{p}$ \\
\hline Cancellation rate (\%) & 7.5 & 9.8 & 2.9 & $<0.02^{2,3}$ \\
\hline Total FSH dose (IU) & $2339 \pm 1248$ & $3298 \pm 1404$ & $2537 \pm 1090$ & $<0.001^{1,3}$ \\
\hline N. of retrieved oocytes & $9.4 \pm 4.3$ & $8.2 \pm 5.3$ & $9.6 \pm 4.0$ & $<0.03^{3}$ \\
\hline MIl oocytes (\%) & 71.2 & 68.8 & 66.9 & ns \\
\hline Ovarian sensitivity & $5.6 \pm 3.2$ & $3.5 \pm 3.5$ & $5.0 \pm 3.0$ & $<0.001^{1,3}$ \\
\hline \multicolumn{5}{|l|}{ Type of treatment (\%) } \\
\hline IVF & 77 & 72 & 68 & ns \\
\hline ICSI & 19 & 23 & 25 & ns \\
\hline Combined & 4 & 5 & 7 & ns \\
\hline Fertilization rate (\%) & 67.7 & 73.4 & 70.2 & ns \\
\hline Cycles with no fertilization (\%) & 6.6 & 8.2 & 10.0 & $<0.04^{2}$ \\
\hline Number of embryos transferred & $2.0 \pm 0.5$ & $2.1 \pm 0.6$ & $2.2 \pm 0.4$ & ns \\
\hline Pregnancy rate/ started cycle (\%) & 41.5 & 36.6 & 35.0 & ns \\
\hline Pregnancy rate/OPU (\%) & 45.0 & 40.6 & 36.1 & ns \\
\hline Pregnancy rate/ ET (\%) & 48.4 & 44.1 & 40.1 & ns \\
\hline Implantation rate (\%) & 24.2 & 24.6 & 22.1 & ns \\
\hline Live-birth rate/ET (\%) & 34.6 & 25.8 & 30.8 & ns \\
\hline
\end{tabular}

${ }^{1}$ Group A vs Group B, ${ }^{2}$ Group B vs Group C, ${ }^{3}$ Group A vs Group C, ns: not significant.

disease and the pregnancy rate [4]. However, several subsequent studies, including a large epidemiological survey [9], reported similar IVF outcome in patients with ovarian endometriosis as in women with other infertility causes $[6,8,26,27]$. A recent study comparing patients with endometriomas with women with nonendometriotic ovarian cysts suggested that ovarian endometriosis was associated with poorer embryo quality, although the pregnancy rate was unaffected [28]. Intervention studies investigating the effectiveness of laparoscopic removal of ovarian endometriosis as a tool to improve subsequent IVF results also show mainly negative results. Several reports showed that the outcome of IVF in patients previously submitted to laparoscopic stripping of endometriomas was similar to that of endometriosis-free controls [27,29-32]. As these studies did not include a group of patients with endometriomas who had not been subject to surgery, the impact of operating ovarian endometriosis per se could not be evaluated. In line with our results, a recent metanalysis showed that the outcome of IVF was similar in patients with in situ ovarian endometriomas as in endometriosisfree women [7]. In 30 infertile patients that served as their own controls as they were treated with IVF both before and after surgical treatment of ovarian endometriosis, Shahine [15] showed that embryo quality on day 3 was not improved after ovarian surgery, and IVF results remained comparable to those obtained before the operation. A recent meta-analysis including five studies comparing surgery vs. no treatment of endometrioma before IVF showed that there was no significant difference in the clinical pregnancy rate between the operated and the non-operated patients [13]. Only the study of Barri [33] reported a better IVF outcome in patients with ovarian endometriosis previously submitted to ovarian surgery vs. patients undergoing IVF as the first therapeutic option. Thus, most available data clearly show that surgical management of endometriomas gives no advantage for a subsequent IVF.

Our results showed that women operated for ovarian endometriosis exhibited several markers of a reduced ovarian reserve. Thus, cancellation rates, mean period [34], antral follicle counts and ovarian sensitivity to FSH/hMG were all reduced in the group of operated women. These findings are well in line with previous data. Patients previously submitted to laparoscopic cystectomy required a higher gonadotropin dose to achieve a similar ovarian response [12,16-18] or showed a lower oocyte yield [12,14,32,35-37]. In women operated for a monolateral ovarian endometrioma, it was reported that the operated ovary produced a lower number of follicles than the contralateral $[38,39]$. Indeed a histologically proven loss of functional ovarian tissue close to the cyst was well documented [40]. Moreover, Tinkanen [10] reported that non-operated patients had significantly more embryos and higher pregnancy and live birth rates than operated women. In a prospective, randomized trial, Demirol [12] showed that operated 
women required a higher FSH dose and a longer stimulation, obtained less oocytes and finally had similar IVF outcome as women with in situ endometriomas. Somigliana [14] reported that women operated for bilateral endometriotic ovarian cysts and subsequently submitted to IVF had a higher withdrawal rate for poor response, retrieved less oocytes despite the use of higher doses of gonadotropins, and had significantly lower pregnancy and delivery rates than non-endometriotic, never operated on the ovary, controls.

In conclusion, with the limitations of a retrospective study we show herein that the presence of ovarian endometriosis is not a cause of poorer IVF outcome and that laparoscopic stripping before IVF does not improve outcome. On the contrary, the operation may reduce ovarian reserve and increase the need for exogenous hormones to retrieve an adequate number of oocytes, thus increasing the overall cost of the treatment. Our observations, in line with most recent data, add evidence against laparoscopic ovarian surgery for endometriomas in asymptomatic patients who are candidates for IVF.

\section{Author details}

'LIVET Infertility and IVF Clinic, Torino, Italy. ${ }^{2}$ Reproductive Medicine and IVF Unit, Department of Obstetrical and Gynecological Sciences, University of Torino, OIRM-S, Anna Hospital, Torino, Italy. ${ }^{3}$ Carl von Linne' Clinic, Uppsala, Sweden.

\section{Authors' contributions}

FB, DG and LDP collected the data and provided the first draft of the manuscript. GG participated in the design of the study and performed the statistical analysis. AR and JH conceived of the study, participated in its design and coordination, and helped to draft the manuscript. All authors read and approved the final manuscript.

\section{Competing interests}

The authors declare that they have no competing interests.

Received: 16 February 2011 Accepted: 17 June 2011

Published: 17 June 2011

\section{References}

1. Somigliana E, Vercellini P, Viganó P, Ragni G, Crosignani PG: Should endometriomas be treated before IVF-ICSI cycles? Hum Reprod Update 2006, 12:57-64.

2. Vercellini $P$, Somigliana E, Viganò P, Abbiati A, Barbara G, Crosignani PG: Surgery for endometriosis-associated infertility: a pragmatic approach. Hum Reprod 2009, 24:254-269.

3. Garcia-Velasco JA, Somigliana E: Management of endometriomas in women requiring IVF: to touch or not to touch. Hum Reprod 2009, 24:496-501.

4. Barnhart K, Dunsmoor-Su R, Coutifaris C: Effect of endometriosis on in vitro fertilization. Fertil Steril 2002, 77:1148-1155.

5. Aboulghar MA, Mansour RT, Serour Gl, Al-Inany HG, Aboulghar MM: The outcome of in vitro fertilization in advanced endometriosis with previous surgery: a case-controlled study. Am J Obstet Gynecol 2003, 188:371-375

6. Suzuki T, Izumi S, Matsubayashi H, Awaji H, Yoshikata K, Makino T: Impact of ovarian endometrioma on oocytes and pregnancy outcome in in vitro fertilization. Fertil Steril 2005, 83:908-913.

7. Gupta S, Agarwal A, Agarwal R, Loret de Mola JR: Impact of ovarian endometrioma on assisted reproduction outcomes. Reprod Biomed Online 2006, 13:349-360.
8. Matalliotakis IM, Cakmak H, Mahutte N, Fragouli Y, Arici A, Sakkas D: Women with advanced-stage endometriosis and previous surgery respond less well to gonadotropin stimulation, but have similar IVF implantation and delivery rates compared with women with tubal factor infertility. Fertil Steril 2007, 88:1568-1572.

9. Wright VC, Chang J, Jeng G, Macaluso M: Assisted reproductive technology surveillance-United States, 2005. MMWR Surveillance Summary 2008, 57:1-23, Does laparoscopic excision of endometriotic ovarian cysts significantly affect ovarian reserve? Insights from IVF cycles. Hum Reprod 2003, 18:2450-2453.

10. Tinkanen $\mathrm{H}$, Kujansuu $\mathrm{E}:$ In vitro fertilization in patients with ovarian endometriomas. Acta Obstet Gynecol Scand 2000, 79:119-122.

11. Garcia-Velasco JA, Mahutte NG, Corona J, Zuniga V, Giles J, Arici A, Pellicer A: Removal of endometriomas before in vitro fertilization does not improve fertility outcomes: a matched, case-control study. Fertil Steril 2004, 81:1194-1197.

12. Demirol A, Guven S, Baykal C, Gurgan T: Effect of endometrioma cystectomy on IVF outcome: a prospective randomized study. Reprod Biomed Online 2006, 12:639-643.

13. Tsoumpou I, Kyrgiou M, Gelbaya TA, Nardo LG: The effect of surgical treatment for endometrioma on in vitro fertilization outcomes: a systematic review and meta-analysis. Fertil Steril 2009, 92:75-87.

14. Somigliana E, Arnoldi M, Benaglia L, lemmello R, Nicolosi AE, Ragni G: IVFICSI outcome in women operated on for bilateral endometriomas. Hum Reprod 2008, 23:1526-1530.

15. Shahine LK, Burney RO, Behr B, Milki AA, Westphal LM, Lathi RB: Embryo quality before and after surgical treatment of endometriosis in infertile patients. J Assist Reprod Genet 2009, 26:69-73.

16. Huang HY, Lee CL, Lai YM, Chang MY, Chang SY, Soong YK: The outcome of in vitro fertilization and embryo transfer therapy in women with endometriosis failing to conceive after laparoscopic conservative surgery. J Am Ass Gynecol Laparosc 1997, 4:299-303.

17. Yazbeck C, Madelenat P, Sifer C, Hazout A, Poncelet C: Ovarian endometriomas: Effect of laparoscopic cystectomy on ovarian response in IVF-ET cycles. Gynecol Obstet Fertil 2006, 34:808-812.

18. Duru NK, Dede M, Acikel CH, Keskin U, Fidan U, Baser I: Outcome of in vitro fertilization and ovarian response after endometrioma stripping at laparoscopy and laparotomy. J Reprod Med 2007, 52:805-809.

19. Ubaldi F, Wisanto A, Camus M, Tournaye H, Clasen K, Devroey P: The role of transvaginal ultrasonography in the detection of pelvic pathologies in the infertility workup. Hum Reprod 1998, 13:330-333.

20. Yaron Y, Peyser MR, Samuel D, Amit A, Lessing JB: Infected endometriotic cysts secondary to oocyte aspiration for in-vitro fertilization. Hum Reprod 1994, 9:1759-1760.

21. Younis JS, Ezra Y, Laufer N, Ohel G: Late manifestation of pelvic abscess following oocyte retrieval, for in vitro fertilization, in patients with severe endometriosis and ovarian endometriomata. J Assist Reprod Genet 1997, 14:343-346.

22. Isaacs JD Jr, Hines RS, Sopelak VM, Cowan BD: Ovarian endometriomas do not adversely affect pregnancy success following treatment with in vitro fertilization. J Assist Reprod Genet 2000, 14:551-553.

23. Mesogitis S, Daskalakis G, Pilalis A, Papantoniou N, Thomakos N, Dessipris N, Koutra $P$, Antsaklis A: Management of ovarian cysts with aspiration and methotrexate injection. Radiology 2005, 235:668-673.

24. Koike T, Minakami H, Motoyama M, Ogawa S, Fujiwara H, Sato I: Reproductive performance after ultrasound-guided transvaginal ethanol sclerotherapy for ovarian endometriotic cysts. Eur J Obstet Gynecol Reprod Biol 2002, 105:39.

25. Yazbeck C, Madelenat $P$, Ayel JP, Jacquesson L, Bontoux LM, Solal P, Hazout A: Ethanol sclerotherapy: a treatment option for ovarian endometriomas before ovarian stimulation. Reprod Biomed Online 2009, 19:121-125.

26. Hickman TN: Impact of endometriosis on implantation. Data from the Wilford Hall Medical Center IVF-ET Program. J Reprod Med 2002, 47:801-808.

27. Marconi G, Vilela M, Quintana R, Sueldo C: Laparoscopic ovarian cystectomy of endometriomas does not affect the ovarian response to gonadotropin stimulation. Fertil Steril 2002, 78:876-878.

28. Kumbak B, Kahraman S, Karlikaya G, Lacin S, Guney A: In vitro fertilization in normoresponder patients with endometriomas: comparison with basal simple ovarian cysts. Gynecol Obstet Invest 2008, 65:212-216. 
29. Al-Azemi M, Bernal AL, Steele J, Gramsbergen I, Barlow D, Kennedy S: Ovarian response to repeated controlled stimulation in in-vitro fertilization cycles in patients with ovarian endometriosis. Hum Reprod 2000, 15:72-75.

30. Canis M, Pouly JL, Tamburro S, Mage G, Wattiez A, Bruhat MA: Ovarian response during IVF-embryo transfer cycles after laparoscopic ovarian cystectomy for endometriotic cysts of $>3 \mathrm{~cm}$ in diameter. Hum Reprod 2001, 16:2583-2586.

31. Donnez J, Wyns C, Nisolle M: Does ovarian surgery for endometriomas impair the ovarian response to gonadotropin? Fertil Steril 2001, 76:662-665.

32. Pabuccu R, Onalan G, Goktolga U, Kucuk T, Orhon E, Ceyhan T: Aspiration of ovarian endometriomas before intracytoplasmic sperm injection. Fertil Steril 2004, 82:705-711.

33. Barri PN, Coroleu B, Tur R, Barri-Soldevila PN, Rodriguez I: Endometriosisassociated infertility: surgery and IVF, a comprehensive therapeutic approach. Reprod Biomed Online 2010, 21:179-185.

34. Brodin T, Bergh T, Berglund L, Hadziosmanovic N, Holte J: Menstrual cycle length is an age-independent marker of female fertility: results from 6271 treatment cycles of in vitro fertilization. Fertil Steril 2008 90:1656-1661.

35. Geber S, Ferreira DP, Spyer Prates LF, Sales L, Sampaio M: Effects of previous ovarian surgery for endometriosis on the outcome of assisted reproduction treatment. Reprod Biomed Online 2002, 5:162-166.

36. Suganuma N, Wakahara Y, Ishida D, Asano M, Kitagawa T, Katsumata Y, Moriwaki T, Furuhashi M: Pretreatment for ovarian endometrial cyst before in vitro fertilization. Gynecol Obstet Invest 2002, 54(Suppl 1):36-40.

37. Loo TC, Lin MY, Chen SH, Chung MT, Tang HH, Lin LY, Tsai YC: Endometrioma undergoing laparoscopic ovarian cystectomy: its influence on the outcome of in vitro fertilization and embryo transfer (IVF-ET). J Assist Reprod Genet 2005, 22:329-333.

38. Ho HY, Lee RK, Hwu YM, Lin MH, Su JT, Tsai YC: Poor response of ovaries with endometrioma previously treated with cystectomy to controlled ovarian hyperstimulation. J Assist Reprod Genet 2002, 19:507-511.

39. Somigliana E, Ragni G, Benedetti F, Borroni R, Vegetti W, Crosignani PG: Does laparoscopic excision of endometriotic ovarian cysts significantly affect ovarian reserve? Insights from IVF cycles. Hum Reprod 2003, $18: 2450-2453$

40. Muzii L, Bellati F, Bianchi A, Palaia I, Manci N, Zullo MA, Angioli R, Panici PB: Laparoscopic stripping of endometriomas: a randomized trial on different surgical techniques. Part II: pathological results. Hum Reprod 2005, 20:1987-1992.

doi:10.1186/1477-7827-9-81

Cite this article as: Bongioanni et al: Ovarian endometriomas and IVF: a retrospective case-control study. Reproductive Biology and Endocrinology 2011 9:81.

\section{Submit your next manuscript to BioMed Central and take full advantage of:}

- Convenient online submission

- Thorough peer review

- No space constraints or color figure charges

- Immediate publication on acceptance

- Inclusion in PubMed, CAS, Scopus and Google Scholar

- Research which is freely available for redistribution 